\title{
Accuracy estimation of mill pin machining with desk-side tools
}

\author{
Julia Anatolievna Bondarenko \\ Belgorod State Technological University after V.G. \\ Shukhov \\ Belgorod, Russia \\ Kdsm2002@mail.ru
}

\author{
Tamara Mikhailovna Sanina \\ Belgorod State Technological University after V.G. \\ Shukhov \\ Belgorod, Russia
}

\author{
Olga Vasilyevna Bestuzheva \\ Belgorod State Technological University after V.G. Shukhov \\ Belgorod, Russia
}

\begin{abstract}
Building materials equipment repair and reconstruction are a challenge as this equipment has large overall dimensions and masses. Requirements to desk side tools precision are expressed in the following: basing surface accuracy of the restored workpiece by the tools; accuracy of working tools movement; basing surface position relative to the guiding support; kinematic chains accuracy; locating accuracy.
\end{abstract}

Keywords-accuracy, machine-tool, form, deviation, parameters, model, system.

\section{INTRODUCTION}

Desk-side tools machining and reconstruction processes are different from that ones by common machine-tools standing on the base. Desk-side tools are put on to the machined detail or near it but placing is done while the tool is put onto the detail, which is a crucial difference between stationary tools and desk-side ones [14-16].

\section{INFORMATIVEPART}

To estimate the accuracy of a large-dimensional detail machining with desk side tools, if deviations in position of nodes and desk-side tools elements, caused by different reasons, are considered as input data, and output parameters are sizing errors, position and form, received during surface machining, then the mathematical model of desk-side tool formation can be represented as follows [9, 10]:

- formation layer, including list of numbers, generalized coordinates of shape-generating system links movements and information about a shape-generating structure. If matrix of these data multiplies by a radius-vector of the cutting tool, then we get a vector equation of the shape formatting;

- shape-generating function flows out of a shapegenerating code, this function relates with tool accuracy balance, which follows from error source and errors in shapegenerating system links position;
- tool accuracy balance in its turn is related to the machined surface, which is formed basing on the specified machined surface;

- Machined surface forms the base surface, which gives size and position errors (deviations);

- form deviation will appear from the base surface data and tool accuracy balance.

If we know system disturbance values their direction and the shape-generating function we can identify desk-side tool accuracy balance. Tool accuracy balance serves as a base to characterize machining accuracy. The base is drawn according to the points of the machined surface; it should coincide with the true surface prescribed by the draw. In such a way, tool output accuracy model is based on calculations and further approximation of the machined surface to the prescribed base surface. Base surface deviations from the nominal ones characterize size and position errors. Desk-side tool formgenerating system contains elements providing mutual position and translation of mechanisms, which go along the prescribed trajectory of the cutting tool relative to the reconstructed detail. Hence, the desk-side tool should have the system of supports for every link to provide the prescribed accuracy. The characteristic of the desk-side tools is that during machining only one tool is applied. As the result, amount of tool shape-generating points is an input signal and the machined surface is an output signal of the shapegenerating accuracy.

During mill pins machining it is necessary to estimate tool output accuracy. Determining of tool accuracy balance is a general problem which can be solved having all information about tool input errors.

But during manufacture and utilization of desk-side tools, it is impossible to receive precise information. So the best way here is to estimate the tool according to the output deviations (errors) of the machined detail. 
To do this it is necessary to determine known functions, determine their number and estimate the parameters, it is necessary to measure many times, on the condition that ( ), that is the number of measurements should be more than the number of components, and function deviation rate should be not less than in $m$ in different points. This problem can be reduced to parameters estimation by linear regression equation [12]:

$$
\begin{gathered}
\Delta_{1}=\Delta\left(u_{1}, v_{1}\right)=a_{11} \delta q_{1}+a_{12} \delta q_{2}+\ldots a_{1 m} \delta q_{m} \\
\Delta_{2}=\Delta\left(u_{2}, v_{1}\right)=a_{21} \delta q_{1}+a_{22} \delta q_{2}+\ldots a_{2 m} \delta q_{m}(1) \\
\Delta_{n}=\Delta\left(u_{n}, v_{n 1}\right)=a_{n 1} \delta q_{1}+a_{n 2} \delta q_{2}+\ldots a_{n m} \delta q_{m}
\end{gathered}
$$

After estimation $\delta q_{j}$ the position of a shape-generating system is changed into $\delta q_{i}$, compensating real machining deviations. If a sporadic error occurs $m=1$, then it is easy to determine its reason and eliminate it. In this case, the inverse problem gives the best tool accuracy estimation.

To estimate size, position accuracy and accuracy of the machined pin surface it is necessary to create a metrological base, that is, to create a base surface. Sizes and base surface position depend on point of deviation of the machined surface from nominal on the type of base surface.

Base surface should be the same as the nominal one, determined by points of the machined surface so that the volume included between the base and the real surfaces should be minimal, all the point of the real surface should be on the same side of the adjoining, that is the nominal surface is determined by the machined surface equation [12].

In this case, a cylindrical pin is machined. All cylindrical surfaces as initial values have radius deviation $\Delta r_{n}$ that is deviation from the nominal radius in the given point.

The equation $\mathrm{r} 0$ of a straight circular cylinder is written:

$$
r_{0}=\left(\begin{array}{l}
R \cos \varphi \\
R \sin \varphi \\
z
\end{array}\right)
$$

These cylinder radius deviations, as projections $\Delta r_{0}$ onto normal to cylindrical surface:

$$
\Delta r_{0}=\left(\begin{array}{l}
\Delta_{1} \cos \varphi-\Delta_{2} \sin \varphi+\Delta_{3} \\
\Delta_{1} \sin \varphi+\Delta_{2} s \cos \varphi+\Delta_{4} \\
\Delta_{5} \\
0
\end{array}\right)
$$

where $\Delta_{1}, \Delta_{2}, \Delta_{3}, \Delta_{4}, \Delta_{5}$ are individual errors of links positions.

The mean square cylinder equation has five parameters: diameter Dsqc and four minor values, they characterize eccentricity and translation of a mean square cylinder relative to $\mathrm{X}$ and $\mathrm{Y}$ coordinate system connected with the nominal cylinder.

Base surface equation is based on nominal

$$
r_{0}=r_{0}\left(u_{1} v_{1} q_{0}\right)
$$

and real

$$
r_{0}=r_{0}+\Delta r_{0}=r\left(u_{1} v_{1} q_{0}\right)
$$
done:

Basing on these equations the base surface equation is

$$
r_{b}=r_{0}\left(u_{1} v_{1} q\right)
$$

where $\mathrm{q}$ is a parameter vector of the base surface;

$$
q=\left(q_{1}, q_{2}, \ldots, q_{p}\right)^{t}
$$

As base surface deviations are little to nominal the base surface equation can be written as [12]:

$$
r_{b}=r_{0}+\Delta r_{b}
$$

where $\Delta r_{b}$ is determined as the total of position and size deviation vectors:

$$
\Delta r_{b}=\varepsilon_{b} r_{0}+d r_{0}
$$

where $\varepsilon_{b}$ is coordinate system position deviation matrix (4x4), connected with base surface, relative to the system of the prescribed equation (5). As these deviations are small $d r_{0}$ there is a total differential of the radius-vector $\mathrm{r} 0$, drawn on all vector components q0. As deviation $\Delta r_{b}$ is small, there is total differential rb drawn on vector components q so we can write $[12,13]$ :

$$
\Delta r_{b}=G \Delta q
$$


Where $\mathrm{G}$ is matrix $4 \times 4$, composed of partial derivatives of column vectors $\partial r_{b} / \partial q_{i}=\partial \Delta r_{b} / \partial \Delta q_{i} ; \quad \Delta q$ is a p-sized column vector composed of position and size deviations $\Delta q_{i}$.

The parameters for the mean square base surface are drawn from the condition of minimal sum of squared deviations of the real surface to base one $r b$, then we can write $[1,12,13]$ :

$$
H \Delta q=d
$$

where $\mathrm{H}$ is matrix pxp with elements $h_{k i}=\int_{s} f_{k} f_{i} d s$, $\mathrm{d}$ is a vector of order $\mathrm{p}$ with elements: $d_{i}=\int_{s} f_{i} \Delta r_{n} d s$, where $\mathrm{fk}$, fi are $\mathrm{k}^{\text {th }}$ and $\mathrm{i}^{\text {th }}$ coordinate $\mathrm{f}$ of normal coefficients $(\mathrm{k}, \mathrm{i}=1,2, \ldots, \mathrm{p}) ; \mathrm{f}=\mathrm{Gtn} ; \mathrm{n}$ is a unit normal vector to the surface $\mathrm{r} 0$.

To construct base cylindrical surface let's determine elements of formula 9 .

By the equation of the quadric cylinder (1) vector $\mathrm{q} 0$ consists of one element $\mathrm{q} 01-$ cylinder radius value $\mathrm{x}=\mathrm{R}$, as $\varphi$ and $\mathrm{z}$ are independent variables $[1,12,13]$.

So, $\mathrm{m}=1, \mathrm{q} 0=\mathrm{q} 01=\mathrm{R}$, then:

$$
d r_{0}=\frac{d r_{0}}{d R} \Delta R=\left(\begin{array}{l}
\cos \varphi \Delta R \\
\sin \varphi \Delta R \\
o \\
o
\end{array}\right)
$$

As $\varphi$ and $\mathrm{z}$ are independent variables, then in the matrix (4x4) $\varepsilon_{b}$ coordinate system position deviation, connected with the base surface, relative to the system specified by the equation (5):

$$
\varepsilon_{b}=\left(\begin{array}{cccc}
0 & -\gamma_{b} & \beta_{b} & \delta_{x b} \\
\gamma_{b} & 0 & -\alpha_{b} & \delta_{y b} \\
-\beta_{b} & \alpha_{b} & 0 & \delta_{z b} \\
0 & 0 & 0 & 0
\end{array}\right)
$$

As deviations along coordinates $\varphi$ are little and $\mathrm{z}$ can be $\delta_{z b}=\gamma_{b}=0$, hence, $\mathrm{n}=4$ and then:

$$
\varepsilon_{b}=\left(\begin{array}{cccc}
0 & 0 & \beta_{b} & \delta_{x b} \\
0 & 0 & -\alpha_{b} & \delta_{y b} \\
-\beta_{b} & \alpha_{b} & 0 & 0 \\
0 & 0 & 0 & 0
\end{array}\right)
$$

Let's substitute the formula data 9 and 11 in 12 and receive:

$$
\Delta r_{b}=\varepsilon_{b} r_{0}+d r_{0}=\left(\begin{array}{l}
\delta_{x b}+\beta_{b} z+\cos \varphi \Delta R \\
\delta_{y b}-\alpha_{b} z+\sin \varphi \Delta R \\
-\beta_{b} R \cos \varphi+\alpha_{b} R \sin \varphi \\
0
\end{array}\right)
$$

Hence, function $\Delta r_{b}$ is the function of two independent $\varphi$ and $\mathrm{z}$ and five small deviations $\delta_{x b}, \delta_{y b}, \alpha_{b}, \beta_{b}, \Delta R$. To receive formulas for calculation $\Delta r_{b}\left(r_{b}\right)$ and if the expression $\Delta r_{0}$ is known through individual errors by formula (3) and literal expression (14) for rb. For this, we should find 25 matrix elements $\mathrm{H}$ and 5 vector elements $\mathrm{d}$, calculate $\Delta q$ after that from the system of equations (11).

Matrix components $\mathrm{H}$ are determined by formula:

$h_{11}=\int_{s}\left(f_{1}\right)^{2} d s=\int_{0}^{2 \pi L} \int_{0}^{2} R \cos ^{2} \varphi d z d \varphi=\pi L R$,

$$
h_{12}=h_{21}=\int_{s} f_{1} f_{2} d s=\int_{0}^{2 \pi L} \int_{0}^{2} R \cos \varphi \sin \varphi d z d \varphi=0,
$$

Where $\mathrm{L}$ is the cylinder length.

$$
H=\frac{1}{6} \pi L R\left(\begin{array}{ccccc}
6 & 0 & 0 & 3 L & 0 \\
0 & 6 & -3 L & 0 & 0 \\
0 & -3 L & 2 L^{2} & 0 & 0 \\
3 L & 0 & 0 & 2 L^{2} & 0 \\
0 & 0 & 0 & 0 & 12
\end{array}\right)
$$

Components $\mathrm{di}$ of the vector $\mathrm{d}$ in the equation 11 are calculated by formula:

$$
d_{i}=\int_{0}^{2 \pi} \int_{0}^{L} R f_{i} \Delta r_{n} d z d \varphi, i=1,2, \ldots, 5
$$




$$
\begin{aligned}
& \delta_{x b}=\frac{2}{\pi L R}\left(2 d_{1}-\frac{3}{L} d_{4}\right) \\
& \delta_{y b}=\frac{2}{\pi L R}\left(2 d_{2}+\frac{3}{L} d_{3}\right) \\
& \alpha_{b}=\frac{2}{\pi L^{3} R}\left(\frac{2}{L} d_{3}+d_{2}\right) \\
& \beta_{b}=\frac{6}{\pi L^{3} R}\left(\frac{2}{L} d_{4}-d_{1}\right) \\
& \Delta R=\frac{d_{3}}{2 \pi L R}
\end{aligned}
$$

\section{CONCLUSION}

The received formulas allow determining size and position deviations, appearing due constant displacement of the cutting tool end in the surface plane.

Shape-generation analysis has proved that a desk-side tool can be placed in any suitable position on the condition that detail axis of rotation coincides with the cutting tool motion line. In this case, shape-generation can be shown as the rotation of curve line segments around machined surfaces axes.

The tests revealed factors appearing during utilization, which influence rotating surfaces shape-generation, their numerical impact on the detail rotation axis displacement and methods of mathematical calculation and numerical impact on the detail rotation axis displacement have been developed.

\section{Acknowledgement}

The article was prepared within development program of the Flagship Regional University on the basis of Belgorod State Technological University named after V.G. Shoukhov, using equipment of High Technology Center at BSTU named after V.G. Shoukhov.

\section{References}

[1] A.N. Avdulov, V.G. Shuster, Mean square base surface construction for arbitrary shaft surface form deviation estimation, Accuracy automatic control on metal-cutting tools, L.: LHSTP, pp.19-24, 1981.

[2] F.G. Banit, Concrete plants equipment maintenance: the textbook, Issue $2^{\text {nd }}$, republished, M.: Gosstroiizdat, 1958.

[3] F.G. Banit, G.S. Krizhanovskiy, B.I.Yakubovich, Utilization, repair and equipment assembly for building materials industry: the textbook for vocational schools in building materials industry, M. : Stroiizdat, 1971.

[4] F.G. Banit, O.A. Nivizhskiy, Mechanical equipment for cement plants: the textbook for vocational schools in building materials industry - Issue $2^{\text {nd }}$ republished and added, M. : Machine-building, 1975.

[5] F.G. Banit, B.I.Yakubovich, Utilization, repair and equipment assembly for building materials industry: the textbook for vocational schools, F-M.: Stroiizdat, 1964.

[6] A.I. Boganov, Cement plants mechanical equipment: the study aid for technical specialities of chemical-technological universities and departments, M. : Mashgiz, 1961.

[7] N.Ye. Drozdov, Utilization, repair and building materials equipment trial: the textbook for universities "Mechanical equipment of plants for building materials, products and constructions"- M.: High School, 1979. $-312 \mathrm{p}$.

[8] N.Ye. Drozdov, M.Ya. Sapozhnikov, Repair and building materials equipment installation for plants: the textbook for universitie, M.: Stroiizdat, 1967.

[9] N.Ye. Drozdov, Utilization and repair of machines for aggregates plants: the study aid for universities, M. : Stroiizdat, 1969.

[10] A.Yu. Albagachiev, S.K. Ambrosimov, O.B. Bavykin, A.N. Bolshakov, Yu.A. Bondarenko, O.F. Viachslavova, Yu.I. Yeremenko, D.M. Zabelyan, S.A. Zaitsev, M.A. Kozlova, Ye.Yu. Krupenya, V.A. Lebedev, I.Ye. Lobanov, A.V. Morozova, N.A. Pelipenko, V.A. Pukhalskii, L.A. Ribak, S.N. Sanin, T.M. Sanina, A.G. Serebrennikova. State-of -the -art machine-building technologies, equipment and tools, Moscow, 2015.

[11] S.K. Ambrosimov, Yu.A. Bondarenko, A.S. Vereshaka, A.D. Verkhoturov, A.V. Kirichek, A.M. Kozlov, Ye.V.Kovaleva, I.Ye Lobanov, G.Ye. Malutin, Ye.B. Mokritskaya, B.Ya. Mokritskii, A.V. Morozova, A.A. Musaev, M.N. Nagorkin, V.V. Nagorkin, L.A. Ribak, P.A. Sablin, G.M. Sevastianov, M.A. Fedorenko, V.P. Fedorov, State-of -the -art machine-building technologies, equipment and tools, Joint monograph, Moscow, 2016.

[12] D.N. Reshetov, V.T. Portman, Cutting tools accuracy, M.: Machinebuilding, 1986.

[13] V.I. Samul, Elasticity and plasticity theory fundamentals, Ways of large-dimensional shafts machining without their dismounting., M.: High School, 1982.

[14] Yu.A. Bondarenko, M.A. Fedorenko, Patent RUS 2242346, 05.05.2003

[15] M.A. Fedorenko, Mechanical treatment of larg -dimensional rotational surfaces without their dismounting in utilization conditions. Machinebuilding technologies, Vol. 10, pp. 14-16, 2008.

[16] M.A. Fedorenko, Yu.A. Bondarenko, T.M. Fedorenko, Providing the required surface roughness of large-dimensional rotating details by desk-side tools, Bulletin of Belgorod State technological University named after V. G. Shukhov, Vol. 2, pp. 35-38, 2008. 